\title{
FAR-INFRARED RADIATION CONTROLLED CHAOS IN n-GaAs
}

\author{
U. Frank, A. Brandl and W. Prettl \\ Naturwissenschaftliche Fakultät II - Physik, \\ Universität, D-8400 Regensburg, Federal Republic of Germany
}

(Recelved 7 November 1988 by M. Cardona)

\begin{abstract}
The effect of far-infrared irradiation on selfgenerated periodic and chaotic current fluctuations in the post-breakdown regimes of $\mathrm{n}-\mathrm{GaAs}$ has been investigated at liquid helium temperature. In an external magnetic field of $40 \mathrm{mT}$ the material showed a sequence of frequency-locked oscillations being ordered according to the Farey-Tree. Depending on the irradiation intensity a shifting and scaling of the bias voltage ranges of the frequency-locking states has been observed.
\end{abstract}

Chaos theory has become one of the most powerful tools in describing the dynamics of nonlinear systems. Besides classical disciplines, nonlinear and chaotic transport phenomena in semiconductors show the wide spectrum of different types of chaotic behavior. These nonlinear spatio-temporal effects have been observed by current fluctuations in Ge [1-6], Si [7], InSb [8] and GaAs [9-12]. Especially the alternating sequence of periodic and chaotic behavior can be treated in terms of the circle-map theory. Although the phenomenological theory sets guidelines for modelling the underlying microscopic mechanisms, the elementary processes causing the observed instabilities are still not well understood.

Previous results obtained from selfgenerated current fluctuations in $\mathrm{n}$-GaAs epitaxial layers, demonstrate the crucial role of an external magnetic field and of the free carrier concentration. For vanishing magnetic field strength only regular relaxation oscillations occur with frequencies up to about $5 \mathrm{MHz}$, depending on the sample and the bias voltage. Applying a magnetic field as weak as $10 \mathrm{mT}$ and varying the bias voltage a sequence of regular oscillations, quasiperiodic behavior showing two independent frequencies, frequency-locking and a Ruelle-Takens-Newhouse scenario have been observed, occuring in well defined voltage ranges $[11,12]$.

In the present study we observed that also far-infrared irradiation of the samples affects the nonlinear dynamics of the electrons in a very sensitive way. Low power FIR irradiation constitutes a linear free carrier generation mechanism, whose rate is easily controllable by varying the intensity, influencing the nonlinear generationrecombination kinetics and transport properties. Our measurements show that the overali qualitative behavior of the time series of the current through the sample and the spectral characteristics of the fluctuations remain unaltered for different control parameter regimes. However, even very low irradiation intensity rescales the voltage ranges of the above mentioned fluctuations and shifts the voltage ranges to higher values. The experimental results will be discussed in terms of the circlemap theory.

The measurements have been carried out on $\mathrm{n}-\mathrm{GaAs}$ epitaxial layers. Samples were mounted in the center of a superconducting solenoid and immersed in liquid helium at $4.2 \mathrm{~K}$. The magnetic field was perpendicular to the current through a sample and to the sample surface, parallel to the [100] crystallographic direction. FIR radiation of $337 \mu \mathrm{m}$ wavelength from a cw $\mathrm{HCN}$ laser was guided by a metallic light pipe homogeneously irradiating the epitaxial layers. The wavevector of the incident radiation was perpendicular to the sample surface. The intensity stability of the laser was better than 2 pc. Several samples were investigated yielding qualitatively the same behavior. Experimental results are reported for an epitaxial layer of $30 \mu \mathrm{m}$ thickness having a donor concentration of $4.5 \times 10^{14} \mathrm{~cm}^{-3}$ and being $78 \mathrm{pc}$ compensated. The electron mobility of the sample was $10^{5} \mathrm{~cm}^{2} / V s$ at $77 \mathrm{~K}$.

Recording the current-voltage characteristic with a load resistor of $1 M \Omega$ shows, that at a critical voltage of about 0.4 volts impact ionization of shallow donors causes a breakdown of the voltage drop at the sample leading to an S-type current-voltage characteristic. Above that critical voltage oscillations and fluctuations of the current arise in distinct voltage intervals. In order to localize the regions in the current-voltage plane where fluctuations occur, the current-voltage characteristics for different FIR powers were recorded by a dynamical method [12], displayed in Fig.1. The hatched areas in the current-voltage plane indicate the extension of fluctuations. Increasing FIR power shifts these areas 


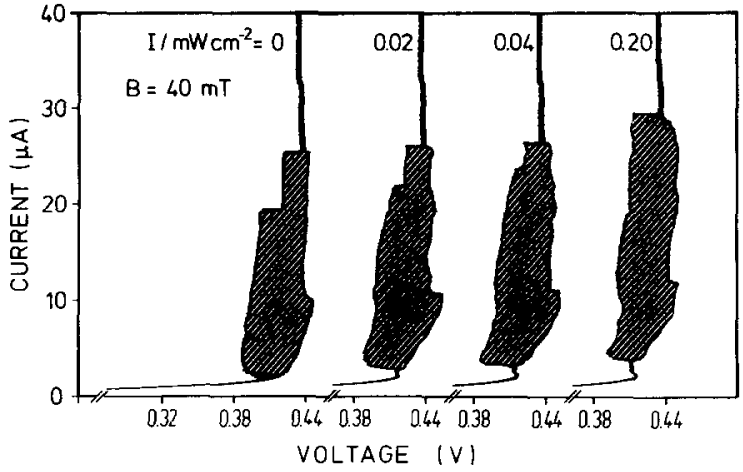

Figure 1: Current-voltage characteristic for various intensities I of FIR exposure, recorded at a fixed external magnetic field $B=40 \mathrm{mT}$. The hatched areas mark the extent of the oscillatory regimes measured with a load resistor of $1 M \Omega$ at a bath temperature of $4.2 \mathrm{~K}$.

to higher currents. These selfgenerated current fluctuations have been analyzed by time series of the voltage drop across the sample. Power spectra were obtained by averaging Fourier transforms of 20 independent time series, consisting of 2048 data points.

In agreement to previous investigations on various samples [12], in the absence of a magnetic field the system is forming coherent oscillations for currents well above the breakdown. Subjecting the sample to a small magnetic field may cause quasiperiodic and frequencylocking behavior. Frequency-locking denotes fixed commensurate ratios of two frequencies $f_{1}$ and $f_{2}$ occuring under variation of a control parameter of the dynamical system, where $f_{1} / f_{2}=q / p$ for small integer values $p$ and q. Here the magnetic field $B$, the FIR-intensity and the voltage over the series combination of the load resistor and the sample act as the accessable external control parameters. In this case the sample was biased in series with a loadresistor of $200 \mathrm{k} \Omega$. Raising the magnetic field strength $\mathrm{B}$ the voltage intervals showing well defined locking states increase [see also 12]. At B $=40 \mathrm{mT}$ a sequence of locking states following the Farey-Treeordering, according to frequency-ratios of $1 / 2,2 / 5,1 / 3$, $1 / 4,2 / 9,1 / 5$ and $1 / 6$ is obtained. Without FIR irradiation locking at $f_{2} / f_{1}=1 / 2$ sets in at the voltage of 0.582 volts $\left(f_{1}=560 \mathrm{kHz}, f_{2}=280 \mathrm{kHz}\right)$. The locked behavior of the two frequencies ends in an $1 / 6$ ratio at a voltage of 0.710 volts $\left(f_{1}=1.230 \mathrm{MHz}, f_{2}=205 \mathrm{kHz}\right)$.

The overlap of two or more locking states on the voltage scale causes a 'frustrated' chaotic behavior [13] of the current fluctuations. Therefore a periodic-chaotic sequence is obtained by increasing the voltage. Figure 2 shows the power spectra of the fluctuations arranged by voltage. Frustrated chaotic regimes are characterized by increased broadband noise in the power spectra. In Fig. 2 proper frequency-locking may be distinguished from chaotic behavior by the existence of dominant peaks in the spectra. The observed frequen-

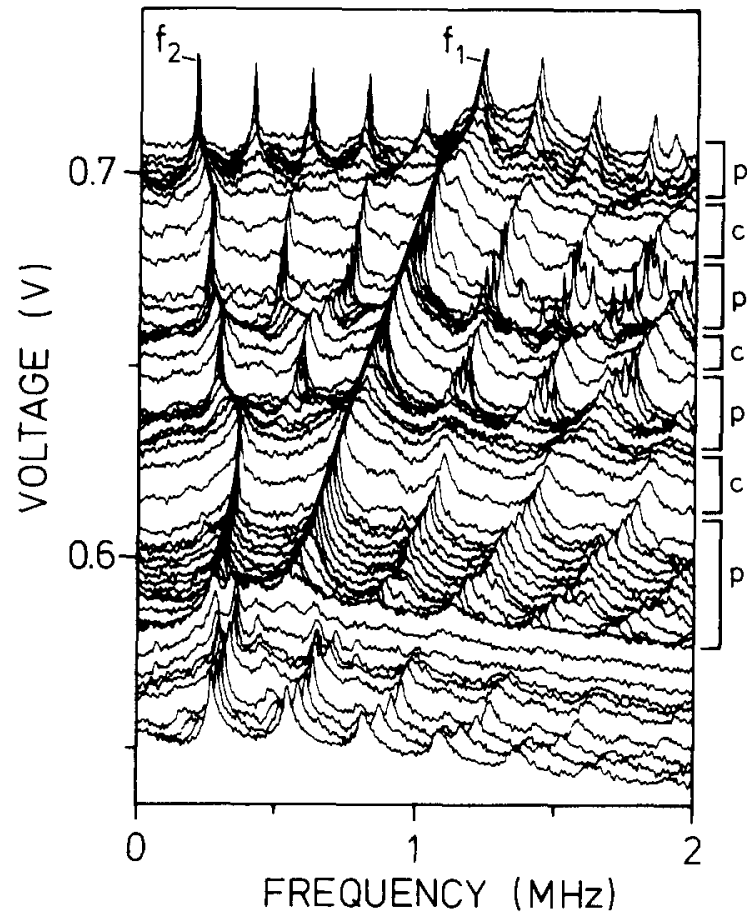

Figure 2: Power spectra of the current fluctuation arranged by the voltage across the series-combination of load resistor and sample. Bold lines are guidelines for the eye indicating both fundamentals $f_{1}$ and $f_{2}$.

cies $f_{1}$ and $f_{2}$ are indicated in Fig.2, in which perodic and chaotic states are marked by $p$ and $c$ respectively. Irradiating the sample by $200 \mu \mathrm{W} / \mathrm{cm}^{2}$, the highest intensity applied in the present study, locking at $f_{2} / f_{1}=1 / 2$ will be achieved at a voltage of 0.68 volts $\left(f_{1}=562 \mathrm{kHz}, f_{2}=281 \mathrm{kHz}\right)$ and the sequence of frequency-locked states ends at 0.773 volts with the ratio $f_{2} / f_{1}=1 / 5\left(f_{1}=1.27 \mathrm{MHz}, f_{2}=254 \mathrm{kHz}\right)$. For all FIR intensities $f_{1}$ increases and $f_{2}$ decreases with rising bias voltage.

In Fig.3 the locking intervals ranging over distinct voltage intervals are indicated and signed by the corresponding locking ratios. From Fig. 3 it is evident that in dependence of weak FIR of $20 \mu W / \mathrm{cm}^{2}, 40 \mu W / \mathrm{cm}^{2}$ and $200 \mu W / \mathrm{cm}^{2}$ the overall structure of the locking states is shifted to bigher voltages. Also the scaling behavior and the degree of overlapping are changed under irradiation. For weak FIR the voltage range in which locking is observed is scaled down. One may depict from Fig. 3 this scaling-down of the locking intervals for weak FIR (Int. $=0.02 \mathrm{~mW} / \mathrm{cm}^{2}$ and $=0.04 \mathrm{~mW} / \mathrm{cm}^{2}$ ). The choosen FIR intensities are too weak to cause significant photoconductivity. For the most extreme case the variation of the sample resistance in the prebreakdown regime is smaller than $0.1 \mathrm{pc}$ at a FIRintensity of $200 \mu \mathrm{W} / \mathrm{cm}^{2}$. Thus the observed shifting and scaling on the voltage scale can not be explained 


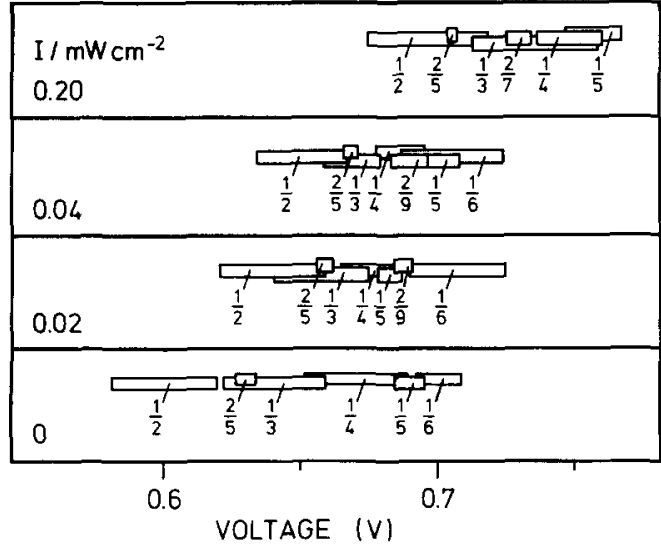

Figure 3: Sequence of frequency-locking states observed within different voltage intervals in dependence of FIR intensitiy $\mathrm{I}$, under constant external magnetic field $\mathrm{B}=40 \mathrm{mT}$. The locking states are marked by the locking ratio $f_{2} / f_{1}$ of the two frequencies $f_{1}$ and $f_{2}$.

by the assumption of a FIR induced change in the internal sample resistances. The observed shifting-up in voltage of the gross oscillatory behavior and the scalingdown of the voltage ranges of frequency-locking on constant rationals demonstrates that far-infrared irradiation does not simply increase the free carrier concentration in thermal equilibrium. Raising the free electron density enhances the impact ionization probability and thus a shifting of the current-voltage characteristics as a whole to lower voltages is expected. This behavior is usually observed by increasing the temperature due to thermal free electron generation. Additionally the circle-map theory suggests a scaling-up of the voltage ranges of mode-locked states as a stronger coupling of the two oscillatory processes may be expected from an increased number of free electrons.

The observed phenomena might be caused by the same microscopic mechanisms which lead to negative far-infrared photoconductivity in $\mathrm{n}-\mathrm{GaAs}$ subjected to a magnetic field [14]. This effect has been explained by the assumption that the free electron concentration is depleted by optically induced free-to-bound transitions from the highly populated $\mathrm{N}=0$ Landau level into donor bound states shifted into the conduction band by the magnetic field. On the other hand impact ionization and subsequent capture of free carriers and oscillatory energy relaxation of free carriers have been assumed to be the cyclic processes leading to the observed quasiperiodic and, depending on the coupling strength of both mechanisms, mode-locking behavior $[12,16]$. Oscillatory energy relaxation is caused by the interplay of the energy loss of high energy electrons due to impact ionization of shallow donors and the acceleration of electrons in the electric field. Electrons scattered to lower energies loose mobility due to enhanced elastic scattering at ionizied impurities. Thus the current through the sample is reduced and the voltage accross the sample increases till the electron system has regained sufficient energy to establish the previous impact ionization rate. Far-infrared radiation at $337 \mu \mathrm{m}$ generates low energy electrons of non-thermal distribution which at first do not contribute to impact ionization however they increase the current and thus reduce the sample voltage. Therefore a higher external bias voltage is necessary to reestablish the impact ionization conditions being realized without irradiation. The gross oscillatory behavior however is not affected by infrared irradiation.

In summary the shifting-up of the frequency-locking behavior of the current fluctuations due to far-infrared irradiation may be understood in terms of existing models of the electron kinetics and non-linear transport. At the present state of investigations it cannot be decided to what extent optical free-to-bound transitions and rescaling of cyclic energy relaxations determine the observed phenomena.

Acknowledgement - We thank E. Bauser, Max-PlanckInstitut für Festkörperforschung, Stuttgart, for provision of the samples. Financial support by the Deutsche Forschungsgemeinschaft is gratefully acknowledged.

\section{REFERENCES}

1. M. Cardona, W. Ruppel: J. Appl. Phys. 31, 1826 (1960).

2. S.B. Bumelione, Yu.K. Pozhela, K.A. Pyragas, A.V. Tamasevicius: Physica 134B, 293 (1985).

3. S.W. Teitsworth, R.M. Westervelt: Physica 23D, 181 (1986), and Phys. Rev. Lett. 56, 516 (1986).

4. J. Peinke, J. Parisi, A. Mühlbach, R.P. Huebener: Z. Naturforsch. 42a, 441 (1987); J. Peinke, J. Parisi, B. Röhricht, K. M. Mayer: Z. Naturforsch. 42a, 841 (1987); U. Rau, J. Peinke, J. Parisi, R.P. Huebener, E. Schöll: Phys. Lett. A 124, 335 (1987), and references herein.
5. E.G. Gwinn, R.M. Westervelt: Phys. Rev. Lett. 57, 1060 (1986), and 59, 157 (1988).

6. G.A. Held, C. Jeffries: Phys. Rev. Lett. 56, 1183 (1986).

7. K. Yamada, N. Takara, H. Imada, N. Miura, C. Hamaguchi: Solid-State Electr. 31, 809 (1988).

8. D.G. Seiler, C.L. Littler, R.J. Justice, P.W. Milonni: Phys. Lett. A 108, 462 (1985).

9. G.N. Maracas, W. Porod, D.A. Johnson, D.K. Ferry, H. Goronkin: Physica 134B, 276 (1985). 
10. K. Aoki, O. Ikezawa, N. Mugibayashi, K. Yamamoto: Physica 134B, 288 (1985), and references herein.

11. A. Brandl, T. Geisel, W. Prettl: Europhys. Lett. 3(4), 401 (1987).

12. J. Spangler, A. Brandl, W. Prettl: Appl. Phys. A 49, to be published.

13. M.H. Jensen, P. Bak, T. Bohr: Phys. Rev. A 30, 1960 (1984).
14. E. Schöll, W. Heisel, W. Prettl: Z. Phys. B 47, 285 (1982).

15. C.J. Stanton, J.W. Wilkins: Phys. Rev. B 35, 9722 (1987).

16. E. Schöll, W. Quade: J. Phys. C: Solid. State Phys. 20, L861-L867 (1987). 